Research Article

\title{
Thermodynamic Properties and Anharmonic Effects in XAFS Based on Anharmonic Correlated Debye Model Debye-Waller Factors
}

\author{
Nguyen Ba Duc $\mathbb{D}^{1},{ }^{1}$ Nguyen Van Hung $\mathbb{D}^{2},{ }^{2}$ Ha Dang Khoa, ${ }^{3}$ Dinh Quoc Vuong, \\ and Tong Sy Tien $\mathbb{C}^{5}$ \\ ${ }^{1}$ Department of Physics, Tan Trao University, Km 6, Trung Mon, Yen Son, Tuyen Quang, Vietnam \\ ${ }^{2}$ Institute of Research and Development, Duy Tan University, 03 Quang Trung, Da Nang, Vietnam \\ ${ }^{3}$ School of Engineering Physics, Hanoi University of Science and Technology, 1 Dai Co Viet, Hanoi, Vietnam \\ ${ }^{4}$ Quang Ninh Education \& Training Department, Cam Pha School, Nguyen Van Cu, Ha Long, Quang Ninh, Vietnam \\ ${ }^{5}$ Department of Basic Sciences, University of Fire Fighting \& Prevention, 243 Khuat Duy Tien, Thanh Xuan, Hanoi, Vietnam
}

Correspondence should be addressed to Nguyen Van Hung; hungnv@vnu.edu.vn

Received 28 June 2017; Revised 14 January 2018; Accepted 7 May 2018; Published 29 July 2018

Academic Editor: Francesco Ruffino

Copyright (c) 2018 Nguyen Ba Duc et al. This is an open access article distributed under the Creative Commons Attribution License, which permits unrestricted use, distribution, and reproduction in any medium, provided the original work is properly cited.

\begin{abstract}
Thermodynamic properties and anharmonic effects in X-ray absorption fine structure (XAFS) have been studied based on the anharmonic correlated Debye model Debye-Waller factors presented in terms of cumulant expansion. The derived analytical expressions of three first XAFS cumulants involve more information on phonon-phonon interactions taken from integration over the first Brillouin zone. Many-body effects are taken into account in the present one-dimensional model based on the first shell near neighbor contributions to the vibrations between absorber and backscatterer atoms. Morse potential is assumed to describe single-pair atomic interaction included in the derived anharmonic interatomic effective potential. The present theory can be applied to any crystal structure including complex systems. Numerical results for $\mathrm{Cu}$ and $\mathrm{Ni}$ are found to be in good agreement with experiment and with those of the other theories.
\end{abstract}

\section{Introduction}

X-ray absorption fine structure (XAFS) has developed into a powerful probe of atomic structure, thermodynamic properties, and anharmonic effects in atomic vibration of substances [1-28]. Thermal atomic vibrations and disorder give rise to Debye-Waller factors (DWFs) in XAFS describing these properties of the considered material. The formalism for including anharmonic effects in XAFS is often based on cumulant expansion [1] where the even cumulants contribute to the amplitude and the odd ones to the phase of XAFS spectra. Hence, the accurate DWFs presented in terms of cumulant expansion are crucial to quantitative treatment of anharmonic XAFS. Consequently, the lack of the precise DWFs or cumulants has been one of the biggest limitations to accurate structural determinations, thermodynamic properties, and anharmonic effects in atomic vibration of materials taken from XAFS experiments. Therefore, a reliable and effective method for treatment of thermal and structural disorders based on DWFs still represents an open problem, whose solution is expected to increase the amount and accuracy of information obtainable from XAFS.

Many efforts have been made to derive procedures for the calculation and analysis of cumulants describing the thermodynamic properties and anharmonic effects or phonon-phonon interactions in temperature-dependent XAFS [2-28] using the classical approach [3-6] and quantum theory [7-28]. Classical theories have the advantages shown by simplicity and possibility of application to high temperatures where anharmonicity is dominant, and 
quantum methods can be applied to both high and low temperatures where quantum effects are evident. The anharmonic correlated Einstein model (ACEM) [9] has been derived for the calculation and analysis of DWFs presented in terms of cumulant expansion up to the third order. Its simplicity and efficiency in XAFS studies is demonstrated by calculating and analyzing XAFS cumulants of fcc [9-13], hcp [14], and crystals and in studying pressure-dependent XAFS [15]. The statistical moment method $[16,17]$ has been applied to calculate the mean square relative displacement (MSRD) including anharmonic contributions of some crystals. The derived pathintegral effective potential (PIEP) method [18] has the advantage for calculating XAFS DWFs presented in terms of cumulant expansion up to the fourth order based on quantum theory including three dimension, correlation, anharmonicity, and many-body effects. The other efforts in XAFS cumulant studies have been shown, for example, by the path-integral Monte Carlo (PIMC) calculations [19], the force constant method (FCM) [20], and the local density approximation (LDA) [21] based on the density functional theory calculations of dynamic matrix.

The purpose of this work is XAFS study of the thermodynamic properties and anharmonic effects of materials based on the anharmonic correlated Debye model (ACDM) DWFs presented in terms of cumulant expansion up to the third order. In Section 2, the analytical expressions for the dispersion relation, correlated Debye frequency and temperature, and three first XAFS cumulants have been derived. They involve more information of phonon-phonon interactions taken from integration over the first Brillouin zone (BZ). The many-body effects are taken into account in the present one-dimensional model based on the first shell near neighbor contributions to the vibrations between absorber and backscatterer atoms. Morse potential is assumed to describe the single-pair atomic interaction included in the derived anharmonic interatomic effective potential. The thermodynamic properties and anharmonic effects in atomic vibration have been analyzed based on the obtained temperature-dependent XAFS cumulants where the anharmonicity causing thermal expansion and the MSRD component perpendicular to bond direction have been discussed in detail. This theory can be applied to any crystal structure including complex systems. Numerical results for $\mathrm{Cu}$ and $\mathrm{Ni}$ presented in Section 3 are compared to a large number of experimental data $[13,19-26]$ and to those calculated using several well-known methods such as ACEM [9], SMM [16], PIEP [18], PIMC [19], FCM [20], and LDA [21] at lowand high temperatures to show the advantage of the present theory. The conclusions on the obtained results are presented in Section 4 of the paper.

\section{Formalism}

2.1. Anharmonic Effective Potential. To determine XAFS cumulants, it is necessary to specify the interatomic interaction potential and force constant [3-27]. Let us consider an anharmonic interatomic effective potential expanded to the third order as follows:

$$
\begin{aligned}
V_{\mathrm{eff}}(x) & \approx \frac{1}{2} k_{\mathrm{eff}} x^{2}+k_{3 \mathrm{eff}} x^{3}, \\
x & =r-r_{0},
\end{aligned}
$$

where $k_{\text {eff }}$ is the effective local force constant, $k_{3 \text { eff }}$ is the cubic effective parameter giving an asymmetry of the anharmonic effective potential, and $x$ is deviation of the instantaneous bond length between two immediate neighboring atoms $r$ from its equilibrium value $r_{0}$.

The anharmonic effective potential (2) is defined based on an assumption in the center-of-mass frame of single bond pair of absorber and backscatterer atoms:

$$
\begin{aligned}
V_{\mathrm{eff}}(x) & =V(x)+\sum_{j \neq i} V\left(\frac{\mu}{M_{i}} x \widehat{R}^{0} \cdot \widehat{R}_{i j}\right), \\
\mu & =\frac{M_{1} M_{2}}{M_{1}+M_{2}},
\end{aligned}
$$

where the first term on the right concerns only absorber and backscatterer with the masses $M_{1}$ and $M_{2}$, respectively, and the second one includes the contributions of their immediate near neighbors to the oscillation between absorber and backscatterer atoms. By projecting such interactions along the bond direction as in (2), the purely one-dimensional model is recovered. Hence, we have extended this effective pair-interaction model to a onedimensional chain to partly account for dispersion effects of the crystals. It is the difference of the present anharmonic effective potential from the single-bond (SB) [7] and singlepair (SP) [8] potentials, which concern only each pair of immediate neighboring atoms given by $V(x)$ without the second one on the right of (2).

Note that the lattice contributions to the oscillation between absorber and backscatterer atoms illustrated by the second term of (2) can be obtained using the first shell near neighbors contributions approach (FSNNCA), which has been successfully applied to bcc crystals [28]. Hence, based on the first shell near neighbor contributions to the vibration between absorber and backscatterer, the many-body effects have been taken into account in the present one-dimensional model.

A Morse potential is assumed to describe the atomic pair-interaction contained in the effective potential (2) and expanded to the third order around its minimum:

$$
V(x)=D\left(e^{-2 \alpha x}-2 e^{-\alpha x}\right) \approx D\left(-1+\alpha^{2} x^{2}-\alpha^{3} x^{3}\right),
$$

where $\alpha$ describes the width of the potential and $D$ is dissociation energy.

Applying Morse potential (3) to (2) and comparing the results to (1), the values of $k_{\text {eff }}$ and $k_{3 \text { eff }}$ of the anharmonic effective potential presented in terms of Morse potential parameters are determined.

2.2. XAFS Cumulants Based on ACDM. In order to include anharmonic effects in the present ACDM, the Hamiltonian of system is written in the summation of the harmonic and cubic anharmonic components, $H_{0}$ and $H_{a}$, respectively: 


$$
H=H_{0}+H_{a},
$$

where $H_{0}$ containing the local force constant, $k_{\text {eff }}$ is used for derivation of second cumulant, and the term $H_{a}$ containing the cubic anharmonic parameter $k_{3 \text { eff }}$ is used for derivation of the first and third cumulants of the materials.

Derivation of the present ACDM is performed using the many-body perturbation approach (MBPA) [29] based on the dualism of an elementary particle in quantum theory, that is, its corpuscular and wave property. Then, we can describe the system in the present ACDM involving all different frequencies up to the Debye frequency as a system consisting of many bodies, that is, many phonons, each of which corresponds to a wave having frequency $\omega(q)$ and wave number $q$ varied in the first BZ. Moreover, based on the FSNNCA only backscattering from the first shell of absorber and backscatterer atoms is taken into consideration. This reduces and simplifies the derivations of the analytical expressions of the considered XAFS cumulants.

For this purpose, the displacement $u_{n}^{\prime} s$ in the parameter $x$ in terms of the displacement of $n$th atom $u_{n}$ of the onedimensional chain described by

$$
x_{n}=u_{n+1}-u_{n},
$$

is related to the phonon displacement operators $A_{q}$ [30] in the form

$$
\begin{aligned}
u_{n} & =\sqrt{\frac{\hbar}{4 N \mu}} \sum_{q} \frac{e^{i q a n}}{\sqrt{\omega(q)}} A_{q^{\prime}}, \\
A_{q} & =A_{-q}^{+}, \\
{\left[A_{q}, A_{q^{\prime}}\right] } & =0 .
\end{aligned}
$$

Then, (5) has resulted as

$$
\begin{gathered}
x_{n}=\sum_{q} e^{i q a n} f(q) A_{q}, \\
f(q)=\sqrt{\frac{\hbar}{4 N \mu \omega(q)}\left(e^{i q a}-1\right),}
\end{gathered}
$$

where $N$ is the atomic number, $\mu$ is reduced mass, and $a$ is the lattice constant.

The frequency $\omega(q)$ contained in (7) and then in all cumulant expressions derived for the oscillation between absorber and backscatterer atoms in XAFS process under the interactions of these atoms with their first shell near neighbors describes the dispersion relation. Using the obtained local force constant, it has resulted as

$$
\omega(q)=\sqrt{\frac{k_{\mathrm{eff}}}{\mu} \mid}\left|\sin \left(\frac{q a}{2}\right)\right|, \quad|q| \leq \frac{\pi}{a} .
$$

At the bounds of the first BZ of the linear chain, $q= \pm \pi / a$, the frequency is maximum so that from (8), we obtain the correlated Debye frequency $\omega_{\mathrm{D}}$ and temperature $\theta_{\mathrm{D}}$ in the form

$$
\begin{aligned}
& \omega_{\mathrm{D}}=\sqrt{\frac{k_{\mathrm{eff}}}{\mu}}, \\
& \theta_{\mathrm{D}}=\frac{\hbar \omega_{\mathrm{D}}}{k_{\mathrm{B}}},
\end{aligned}
$$

where $k_{\mathrm{B}}$ is Boltzmann constant.

Based on the above results, the cubic component of Hamiltonian is expressed as

$$
H_{\mathrm{c}}=k_{3 \mathrm{eff}} x^{3}=\sum_{q_{1}, q_{2}, q_{3}} V\left(q_{1}, q_{2}, q_{3}\right) A_{q_{1}} A_{q_{2}} A_{q_{3}},
$$

or in the following form using (6) for the displacement of $n$th atom

$$
\begin{aligned}
H_{\mathrm{a}}= & k_{3 \mathrm{eff}} \sum_{n}\left(u_{n+1}-u_{n}\right)^{3} \\
= & k_{3 \mathrm{eff}} \sum_{q_{1}, q_{2}, q_{3}}\left(\sum_{n} e^{i\left(q_{1}+q_{2}+q_{3}\right) a n}\right) \\
& \cdot f\left(q_{1}\right) f\left(q_{2}\right) f\left(q_{3}\right) A_{q_{1}} A_{q_{2}} A_{q_{3}} .
\end{aligned}
$$

Comparing (11) to (10) and indicating

$$
\begin{aligned}
& \Delta(q)=\frac{1}{N} \sum_{n} e^{i q n a}, \\
& \Delta(0)=\sum_{n} e^{i 0 n a}=N,
\end{aligned}
$$

with $N$ as the atomic number, we obtain

$$
V\left(q_{1}, q_{2}, q_{3}\right)=k_{3 \mathrm{eff}} \Delta\left(q_{1}+q_{2}+q_{3}\right) f\left(q_{1}\right) f\left(q_{2}\right) f\left(q_{3}\right) .
$$

Using (6) and (12), (13) changes into

$$
\begin{aligned}
V\left(q_{1}, q_{2}, q_{3}\right)= & k_{3 \mathrm{eff}}\left(\frac{\hbar}{4 N \mu}\right)^{3 / 2}\left(\sum_{n} e^{i\left(q_{1}+q_{2}+q_{3}\right) a n}\right) \\
& \cdot \frac{\left(e^{i q_{1} a}-1\right)\left(e^{i q_{2} a}-1\right)\left(e^{i q_{3} a}-1\right)}{\sqrt{\omega\left(q_{1}\right) \omega\left(q_{2}\right) \omega\left(q_{3}\right)}} .
\end{aligned}
$$

In the MBPA [28], the value $\langle x\rangle$ is calculated using the expression

$$
\langle x\rangle=\frac{\sum_{q} f(q)\left\langle A_{q} S(\beta)\right\rangle_{0}}{\langle S(\beta)\rangle_{0}}
$$

$$
\begin{aligned}
S(\beta) & =\sum_{n=0}^{\infty} \frac{(-1)^{n}}{n !} \int_{0}^{\beta} d \tau_{1} \ldots \int_{0}^{\beta} d \tau_{n} T\left[H_{a}\left(\tau_{1}\right) \ldots H_{a}\left(\tau_{n}\right)\right] \\
H_{a}(t) & =e^{t H_{0}} H_{a} e^{-t H_{0}}
\end{aligned}
$$


which takes backscattering only from the first shell.

Substituting into (15) the relations [29]

$$
\begin{aligned}
\left\langle A_{q} S(\beta)\right\rangle_{0} & =-\int d \tau\left\langle T\left[A_{q} \widehat{H}_{1}(\tau)\right]\right\rangle, \\
\left\langle A_{q}\right\rangle_{0} & =0,
\end{aligned}
$$

we obtain

$$
\begin{aligned}
\langle x\rangle= & -\sum_{q} f(q) \sum_{q_{1}, q_{2}, q_{3}} V\left(q_{1}, q_{2}, q_{3}\right) \\
& \cdot \int_{0}^{\beta} d \tau\left\langle T\left[\widehat{A}_{q}(0) \widehat{A}_{q_{1}}(\tau) \widehat{A}_{q_{2}}(\tau) \widehat{A}_{q_{3}}(\tau)\right]\right\rangle_{0} .
\end{aligned}
$$

Using the Wick theorem for T-product in the integral, the harmonic phonon Green function [29]

$$
\begin{aligned}
& G_{q, q^{\prime}}^{0}(\tau)=\left\langle T\left[\widehat{A}_{q}(\tau) \widehat{A}_{q^{\prime}}(0)\right]\right\rangle_{0}, \\
& G_{q, q^{\prime}}^{0}(\tau)=-\delta_{q,-q^{\prime}}\left\{\left\langle n_{q}+1\right\rangle e^{-\hbar \omega(q) \tau}+\left\langle n_{q}\right\rangle e^{\hbar \omega(q) \tau}\right\},
\end{aligned}
$$

the symmetric properties of $V\left(q_{1}, q_{2}, q_{3}\right)$ [30], properties of function $\delta_{q,-q^{\prime}}$, the phonon density

$$
\begin{aligned}
\left\langle n_{q}\right\rangle & =\frac{1}{Z(q)-1}, \\
Z(q) & =\exp (\beta \hbar \omega(q)), \\
\beta & =\frac{1}{k_{\mathrm{B}} T},
\end{aligned}
$$

as well as $\omega(q)$ from (8), $f(q)$ from (7), $\Delta(0)$ from (12) and the phonon momentum conservation in the first BZ, we change (18) into the following:

$$
\begin{aligned}
\langle x\rangle & =-\frac{3 \hbar k_{3 \mathrm{eff}}}{4 N \mu k_{\mathrm{eff}}} \sum_{q} \frac{\left(e^{i q a}-1\right)\left(e^{-i q a}-1\right)}{\omega(q)} \frac{1+Z(q)}{1-Z(q)} \\
& =-\frac{3 \hbar k_{3 \mathrm{eff}}}{N \sqrt{2 \mu k_{\mathrm{eff}}^{3}}} \sum_{q} \sin \frac{q a}{2} \frac{1+Z(q)}{1-Z(q)} .
\end{aligned}
$$

Using this expression, the first cumulant describing the net thermal expansion or lattice disorder in XAFS theory has resulted as

$$
\sigma^{(1)}(T)=\langle x\rangle=\sigma_{0}^{(1)} \int_{0}^{\pi / a} \omega(q) \frac{1+Z(q)}{1-Z(q)} d q=\frac{\sigma_{0}^{(1)}}{\sigma_{0}^{2}} \sigma^{2}
$$

$$
\begin{aligned}
\sigma_{0}^{(1)} & =-\frac{3 a \hbar k_{3 \mathrm{eff}}}{2 \pi k_{\mathrm{eff}}^{2}}, \\
Z(q) & =\exp (\beta \hbar \omega(q)), \\
\beta & =\frac{1}{k_{\mathrm{B}} T},
\end{aligned}
$$

where $\sigma^{2}$ is the second cumulant describing the mean square relative displacement (MSRD) and has the following form:

$$
\begin{aligned}
\sigma^{2}(T) & =\left\langle x^{2}\right\rangle=\sigma_{0}^{2} \int_{0}^{\pi / a} \omega(q) \frac{1+z(q)}{1-z(q)} d q, \\
\sigma_{0}^{2} & =\frac{\hbar a}{2 \pi D k_{\text {eff }}},
\end{aligned}
$$

where using (8) for $\omega(q)$, (7) for $x_{n}$ and $f(q)$, (12) for $\Delta(q)$ and $\Delta(0),(19)$ for $G_{q, q^{\prime}}^{0}(t)$, and (20) for $\left\langle n_{q}\right\rangle$ we calculate $\left\langle x^{2}\right\rangle$

$$
\left\langle x^{2}\right\rangle=\left\langle\sum_{n}\left(x_{n+1}-x_{n}\right)^{2}\right\rangle_{0}=\frac{\hbar}{N \sqrt{2 \mu}} \sum_{q}\left|\sin \frac{q a}{2}\right| \frac{1+Z(q)}{1-Z(q)} \text {. }
$$

The third cumulant is the mean cubic relative displacement (MCRD) describing the asymmetry of the pair distribution function in XAFS theory and has resulted as

$$
\begin{aligned}
\sigma^{(3)}(T) \cong & \left\langle x^{3}\right\rangle-3\left\langle x^{2}\right\rangle\langle x\rangle=\sigma_{0}^{(3)} \int_{0}^{\pi / a} d q_{1} \int_{-\pi / d}^{\pi / a-q_{1}} d q_{2} \frac{\omega\left(q_{1}\right) \omega\left(q_{2}\right) \omega\left(q_{1}+q_{2}\right)}{\omega\left(q_{1}\right)+\omega\left(q_{2}\right)+\omega\left(q_{1}+q_{2}\right)} \\
& \cdot\left\{1+6 \frac{\omega\left(q_{1}\right)+\omega\left(q_{2}\right)}{\omega\left(q_{1}\right)+\omega\left(q_{2}\right)-\omega\left(q_{1}+q_{2}\right)} \frac{e^{\beta \hbar\left[\omega\left(q_{1}\right)+\omega\left(q_{2}\right)\right]}-e^{\beta \hbar \omega\left(q_{1}+q_{2}\right)}}{\left(e^{\beta \hbar \omega\left(q_{1}\right)}-1\right)\left(e^{\beta \hbar \omega\left(q_{2}\right)}-1\right)\left(e^{\beta \hbar \omega\left(q_{1}+q_{2}\right)}-1\right)}\right\}, \\
\sigma_{0}^{(3)}= & \frac{3 \hbar^{2} k_{3 \mathrm{eff}}}{2 N^{2} k_{\mathrm{eff}}^{3}}
\end{aligned}
$$

where the calculation of $\left\langle x^{3}\right\rangle$ is analogous to the one of $\langle x\rangle$ above, that is,

$$
\left\langle x^{3}\right\rangle=\frac{\sum_{q_{1}, q_{2}, q_{3}} f\left(q_{1}\right) f\left(q_{2}\right) f\left(q_{3}\right)\left\langle A_{q_{1}} A_{q_{2}} A_{q_{3}} S(\beta)\right\rangle_{0}}{S(\beta)_{0}},
$$


TABLE 1: Expressions of cumulants in LT $(T \rightarrow 0)$ and HT $(T \rightarrow \infty)$ limits.

\begin{tabular}{lcr}
\hline Cumulant & $T \rightarrow 0$ & $T \rightarrow \infty$ \\
\hline$\sigma^{(1)}$ & $-\left(6 \hbar k_{3 \mathrm{eff}} / \pi \sqrt{2 \mu k_{\mathrm{eff}}^{3}}\right)(1+z)$ & $-\left(3 k_{3 \mathrm{eff}} k_{\mathrm{B}} T / k_{\mathrm{eff}}^{2}\right)$ \\
\hline$\sigma^{2}$ & $\left(2 \hbar / \pi \sqrt{2 \mu k_{\mathrm{eff}}}\right)(1+z)$ & $k_{\mathrm{B}} T / k_{\mathrm{eff}}$ \\
\hline$\sigma^{(3)}$ & $-\left(3 \hbar^{2} k_{3 \mathrm{eff}} / 2 N^{2} k_{\mathrm{eff}}^{3}\right)$ & $-\left(\left(6 k_{3 \mathrm{eff}}\left(k_{\mathrm{B}} T\right)^{2}\right) / k_{\mathrm{eff}}^{3}\right)$ \\
& $\sum_{q_{1}, q_{2}, q_{3}}\left(\omega\left(q_{1}\right) \omega\left(q_{2}\right) \omega\left(q_{3}\right)\right) /\left(\omega\left(q_{1}\right)\right.$ & \\
& $\left.+\omega\left(q_{2}\right)+\omega\left(q_{3}\right)\right)\left(1+Z_{3}\right)$ &
\end{tabular}

$\sigma^{(1)} \sigma^{2} / \sigma^{(3)}$ $-$

where using $S(\beta)$ from (17) with limiting only the cubic anharmonic term, the Wick theorem for T-product, and the symmetric properties of $V\left(q_{1}, q_{2}, q_{3}\right)$ [30], we calculated $\left\langle x^{3}\right\rangle$. The product $3\left\langle x^{2}\right\rangle\langle x\rangle$ has been calculated using $\left\langle x^{2}\right\rangle$ from (24) and $\langle x\rangle$ from (21).

In the above expressions for the cumulants in the present ACDM, $\sigma_{0}^{(1)}, \sigma_{0}^{2}, \sigma_{0}^{(3)}$ are zero-point energy contributions to the first, second, and third cumulant, respectively, and these cumulant expressions have been obtained for the case of large phonon numbers, when the summation over $q$ is replaced by the corresponding integral in the first $\mathrm{BZ}$. Moreover, we have used the phonon momentum conservation in the first $\mathrm{BZ}$ [30] to describe the value of $q_{3}$ by $q_{1}$ and $q_{2}$ for the first and third cumulant. This leads to reducing the integrations for these cumulants given by (22) and (25), respectively.

Note that the present theory is valid for any crystal structure including complex systems; therefore, the other developments for the monatomic [27] and bcc [28] crystals are only its special cases.

\subsection{High-and Low-Temperature Limits of XAFS Cumulants.} It is useful to consider the high-temperature (HT) limit, where the classical approach $[5,6]$ is applicable, and the lowtemperature (LT) limit, where the quantum theory must be used [9]. In the HT limit, we use the approximation

$$
Z(q) \approx 1+\beta \hbar \omega(q)
$$

to simplify the expressions for the cumulants. In the LT limit, $Z(q) \gg 1$, so that all temperature-dependent terms approach zero and the cumulants approach constant values, for example, their zero-point contributions. These results are written in Table 1.where

$$
\begin{aligned}
z & =\frac{1}{a} \int_{0}^{\pi / a} \frac{\sin (q a / 2)}{Z(q)} d q, \\
Z_{3} & =\frac{6\left[\omega\left(q_{1}\right)+\omega\left(q_{2}\right)\right]}{\left[\omega\left(q_{1}\right)+\omega\left(q_{2}\right)\right]^{2}-\omega^{2}\left(q_{3}\right)} \frac{Z\left(q_{3}\right)-Z\left(q_{1}\right) Z\left(q_{2}\right)}{Z\left(q_{1}\right) Z\left(q_{2}\right) Z\left(q_{3}\right)} .
\end{aligned}
$$

Note from Table 1 that at high temperatures, the first and second cumulants are proportional to the temperature $T$, the third cumulant to $T^{2}$ as the standard characters for these quantities as it was mentioned in the other theories [7-9]. At low temperatures, they approach their zero-point energy contributions which also involve contributions of $q$-values from the first BZ. Moreover, at high temperature, the cumulant ratio $\sigma^{(1)} \sigma^{2} / \sigma^{(3)}$ approaches the classical value of $1 / 2[5,6]$.

\section{Numerical Results and Discussions}

Now the expressions derived in the previous section are applied to numerical calculations for $\mathrm{Cu}$ and $\mathrm{Ni}$ in $\mathrm{fcc}$ phase using their Morse potential parameters [31] $D=0.3429 \mathrm{eV}$, $\alpha=1.3588 \AA^{-1}$ for $\mathrm{Cu}$ and $D=0.4205 \mathrm{eV}, \alpha=1.4199 \AA^{-1}$ for $\mathrm{Ni}$, which were obtained using experimental values for the energy of sublimation, the compressibility, and the lattice constant. The values of local force constant $k_{\text {eff }}$, correlated Debye frequency $\omega_{\mathrm{D}}$, and temperature $\theta_{\mathrm{D}}$ calculated using the present theory are given in Table 2 . They are found to be in good and reasonable agreement with the experimental values $[23,25]$, where the experimental values of [23] have been calculated from its measured Morse potential parameters [23] as an extraction method which was used elsewhere [13].

Thermodynamic properties and anharmonic effects of materials depend on the atomic interactions described by the anharmonic interatomic effective potentials $V_{\text {eff }}$ presented in Figure 1. The present ACDM includes dispersion relations $\omega(q)$ (Figure 2) to involve contributions of all atomic vibration frequencies. Their values for $\mathrm{Cu}$ and $\mathrm{Ni}$ calculated using the present theory are shown in good agreement with experiment taken from the measured Morse potential parameters [23]. The anharmonic effective potentials are asymmetric causing the anharmonic shifting, and the correlated frequencies approach the values of Debye frequencies (Table 2) at the bounds of the first BZ, $q= \pm \pi / a$.

Figure 3 shows good agreement of temperature dependence of first cumulant $\sigma^{(1)}(T)$ of $\mathrm{Cu}$ and Ni calculated using the present theory with the experimental values (Expt.) $[13,19,23]$ for $\mathrm{Cu}$ and [23] for Ni. Moreover, the results for $\mathrm{Cu}$ agree well with those calculated using the ACEM [9]. Note that, using the first cumulant, we can obtain temperature dependence of the first shell near neighbor distance based on the expression $R(T)=R(0)+\sigma^{(1)}(T)$. 
TABLE 2: The values of local force constant $k_{\text {eff }}$, Debye frequency $\omega_{\mathrm{D}}$, and temperature $\theta_{D}$ of $\mathrm{Ni}$ and $\mathrm{Cu}$ calculated using the present theory compared to experiment $[23,25]$.

\begin{tabular}{lccc}
\hline Crystal & $k_{\text {eff }}(\mathrm{N} / \mathrm{m})$ & $\omega_{\mathrm{D}}\left(\times 10^{13} \mathrm{~Hz}\right)$ & $\theta_{\mathrm{D}}(\mathrm{K})$ \\
\hline $\mathrm{Ni}$, present & 67.9750 & 5.2637 & 376.00 \\
$\mathrm{Ni}$, expt. [23] & 63.4596 & 5.0881 & 378.00 \\
$\mathrm{Cu}$, present & 50.7181 & 4.3717 & 333.94 \\
$\mathrm{Cu}$, expt. [23] & 50.3450 & 4.3556 & 332.71 \\
$\mathrm{Cu}$, expt. [25] & - & - & 328.00 \\
\hline
\end{tabular}

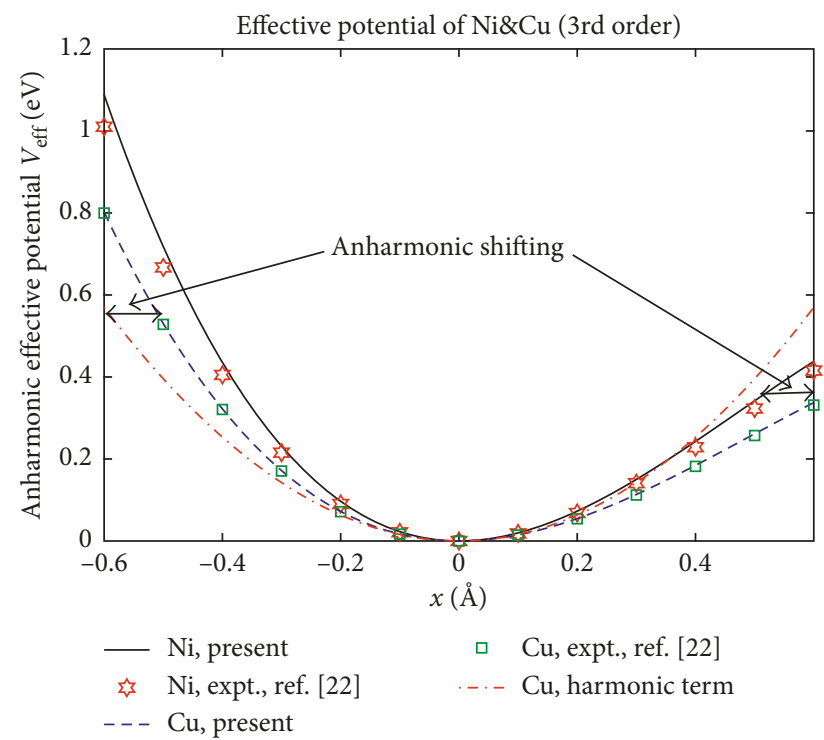

FIgURE 1: Anharmonic interatomic effective potentials $V_{\text {eff }}$ of $\mathrm{Cu}$ and Ni calculated using the present theory compared to the experimental values (Expt.) [23] and to its harmonic term to show its anharmonic shifting.

Temperature dependence of second cumulant $\sigma^{2}(T)$ (Figure 4) of $\mathrm{Cu}$ and $\mathrm{Ni}$ calculated using the present theory agrees well with the experimental values Expt. [13, 22, 23, 25] for $\mathrm{Cu}$ and [23] for $\mathrm{Ni}$, as well as with those calculated using the ACEM [9]. Here, the present result is also compared to those calculated using the SMM [16], FCM [20], and LDA [21] for $\mathrm{Cu}$ and PIEP [18] for $\mathrm{Ni}$, which are found to be in reasonable agreement with the experiment.

Figure 5 shows good agreement of temperature dependence of third cumulant $\sigma^{(3)}(T)$ of $\mathrm{Cu}$ and Ni calculated using the present theory with the experimental values Expt. $[13,23,24,26]$ for $\mathrm{Cu}$ and [23] for Ni. These results are also found to be in good agreement with those calculated using the ACEM [9] and the PIMC [19] for $\mathrm{Cu}$.

The cumulant ratio $\sigma^{(1)} \sigma^{2} / \sigma^{(3)}$ is often considered as a standard for cumulant studies to identify the temperature above which the classical limit is applicable [9-14]. Figure 6 shows that in the present $\mathrm{ACDM}$ this temperature is the Debye temperature $\left(\theta_{\mathrm{D}}=334 \mathrm{~K}\right.$ for $\mathrm{Cu}$ and $376 \mathrm{~K}$ for $\left.\mathrm{Ni}\right)$ while such temperature for ACEM is the Einstein temperature [9] because from this temperature the ratio reaches the classical value of $1 / 2[9,10,14]$ and classical limit is applicable. Here, the result for $\mathrm{Cu}$ agrees well with the experimental values at $300 \mathrm{~K}, 400 \mathrm{~K}$, and $500 \mathrm{~K}$ [13].

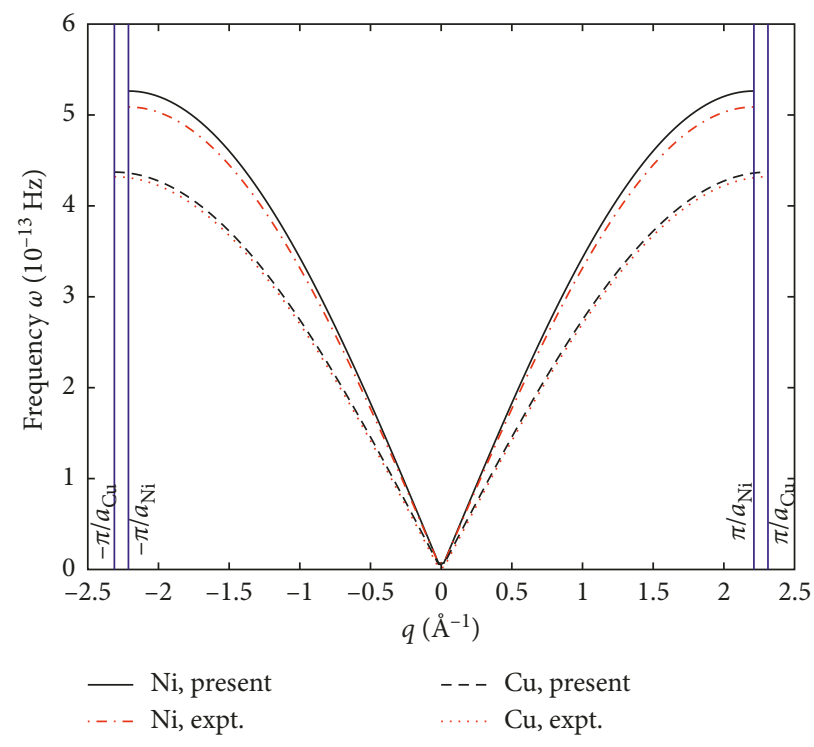

FIgure 2: Dispersion relations $\omega(q)$ of $\mathrm{Cu}$ and Ni calculated using the present theory compared to the experimental values (Expt.) [23].

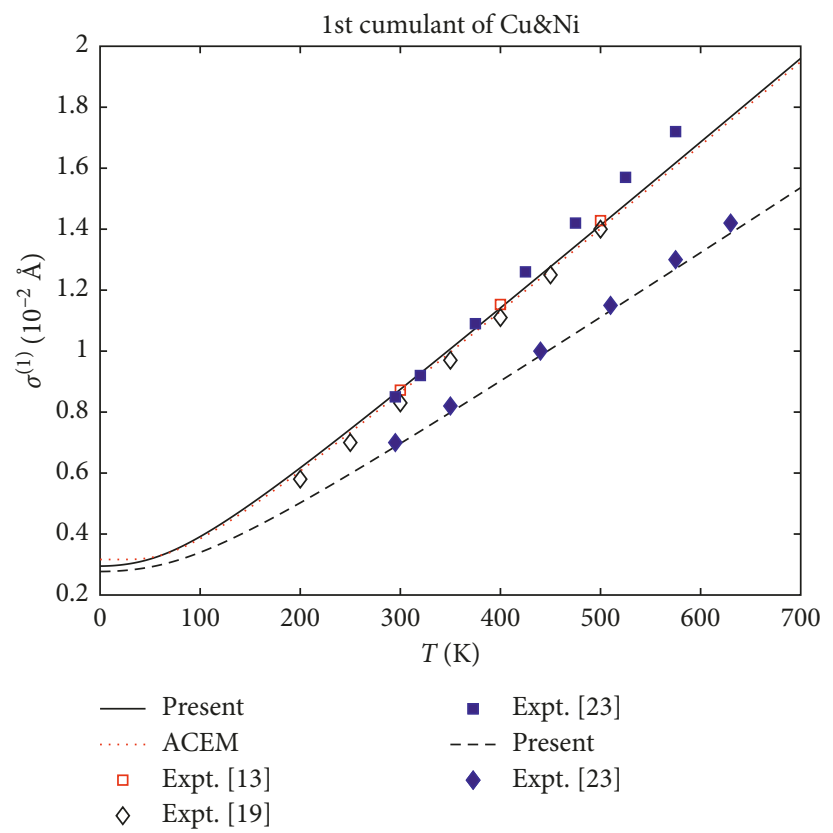

Figure 3: Temperature dependence of first cumulant $\sigma^{(1)}(T)$ of $\mathrm{Cu}$ and $\mathrm{Ni}$ calculated using the present theory compared to the experimental values (Expt.) [13, 19, 23] for $\mathrm{Cu}$ and [23] for $\mathrm{Ni}$, as well as to those calculated using the ACEM [9] for $\mathrm{Cu}$.

Anharmonicity is the result of phonon-phonon interaction and gives rise to thermal expansion shown in temperature-dependent XAFS of materials. The anharmonic effects in XAFS have been detected quite early [32]. After the first pioneering studies on $\mathrm{AgI}$ [33] and $\mathrm{CuBr}$ [2], it has been shown that anharmonicity cannot be neglected in XAFS studies of thermodynamic properties and structural determination of materials [2-28]. The XAFS cumulants parametrize the asymmetric distribution of interatomic 


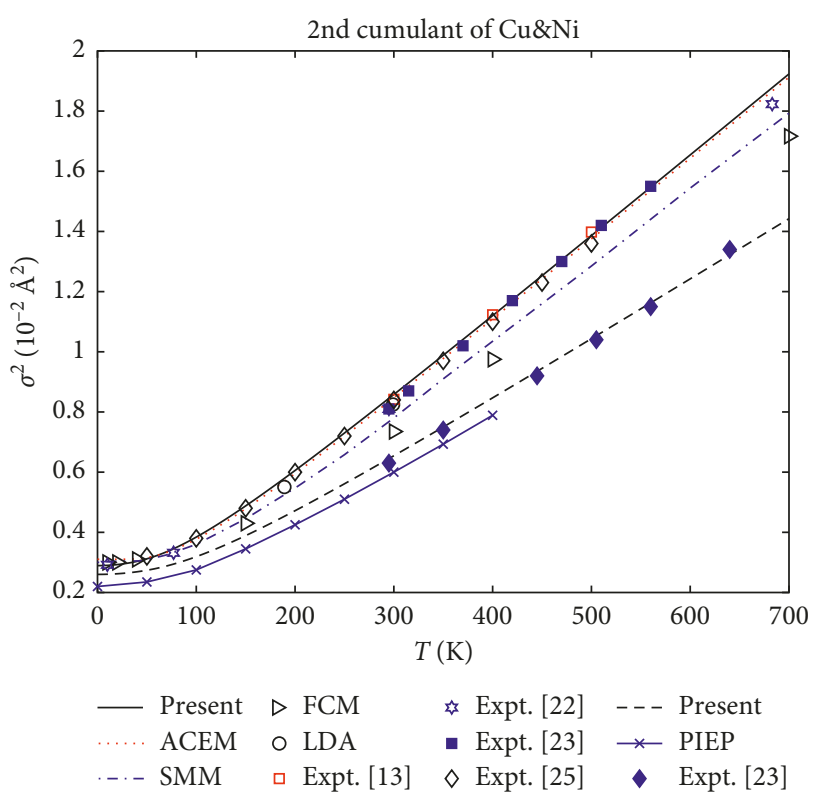

Figure 4: Temperature dependence of second cumulant $\sigma^{2}(T)$ of $\mathrm{Cu}$ and $\mathrm{Ni}$ calculated using the present theory compared to the experimental values (Expt.) $[13,22,23,25]$ for $\mathrm{Cu}$ and [23] for $\mathrm{Ni}$, as well as to those calculated using the ACEM [9], SMM [16], FCM [20], and LDA [21] for $\mathrm{Cu}$ and PIEP [18] for $\mathrm{Ni}$.

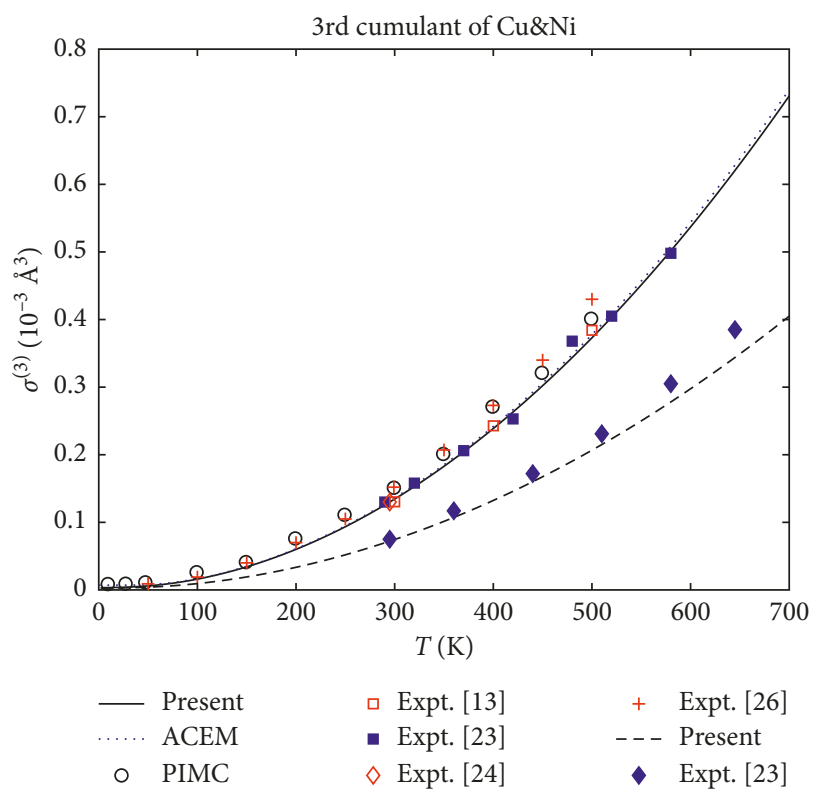

FIgURE 5: Temperature dependence of third cumulant $\sigma^{(3)}(T)$ of $\mathrm{Cu}$ and $\mathrm{Ni}$ calculated using the present theory compared to the experimental values Expt. [13, 23, 24, 26] for $\mathrm{Cu}$ and [23] for $\mathrm{Ni}$, as well as to those calculated using the ACEM [9] and the PIMC [19] for $\mathrm{Cu}$.

distances and can be connected to the force constants of a one-dimensional effective pair potential $[5,9-15,34]$. In particular, the first three cumulants measure the average value, the variance, and the asymmetry of the distribution, respectively [25]. Both the first and third cumulants have often been considered equally sensitive to thermal expansion [35]. This equivalence, which is valid for a one-dimensional

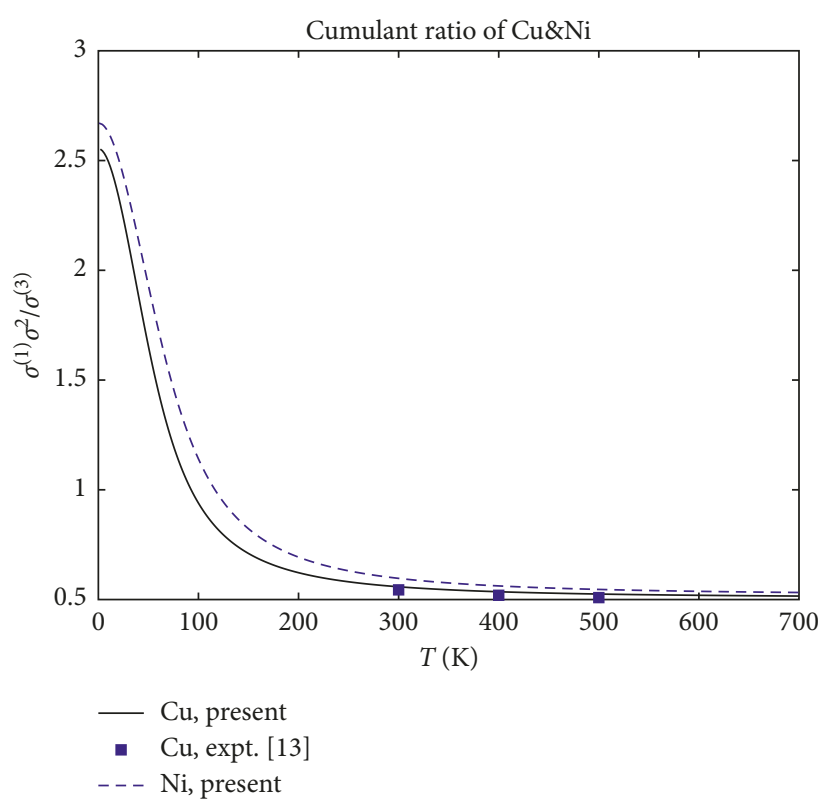

FIGURE 6: Temperature dependence of cumulant ratio $\sigma^{(1)} \sigma^{2} / \sigma^{(3)}$ of $\mathrm{Cu}$ and $\mathrm{Ni}$ calculated using the present theory, where the result for $\mathrm{Cu}$ is compared to the experimental values at $300 \mathrm{~K}, 400 \mathrm{~K}$, and $500 \mathrm{~K}[13]$.

system, where the average distance is solely modified by the asymmetry of the interaction potential, was not confirmed by accurate XAFS measurements of nearest neighbors' distances in several simple crystals $[26,32,36]$. Actually, the first XAFS cumulant is lager than the distance between the centers of the probability distribution functions, owing to the effect of atomic vibrations perpendicular to the bond direction [37, 38], and its temperature dependence is stronger than the thermal expansion measured by Bragg diffraction or by macroscopic techniques. The difference between XAFS and crystallographic thermal expansion can be attributed to the MSRD component perpendicular to the bond direction $[21,26]$. Unfortunately, the PIMC calculations [19] have been performed on $\mathrm{Cu}$. Here, the second cumulant and the parallel MSRD have been independently evaluated from the set of configurations generated by PIMC. The agreement between the two values suggests that the contribution of the perpendicular MSRD to the second cumulant is negligible, at least for $\mathrm{Cu}$. Moreover, the good agreement of cumulants calculated by ACDM in the present work with several experimental data also confirms the above conclusion based on the PIMC calculations.

Consequently, in present work, the thermodynamic properties and anharmonic effects of $\mathrm{Cu}$ and $\mathrm{Ni}$ in the fcc phase have been studied based on the DWFs presented in terms of cumulant expansion. Here, the second cumulant describing MSRD is primarily a harmonic effect. But, the first cumulant describing the net thermal expansion or lattice disorder and the third cumulant or MCRD describing the asymmetry of pair atomic distribution function are entirely anharmonic effects. These obtained quantities are evidently temperature-dependent where the first and second cumulants are proportional to the temperature $T$ and the 
third cumulant is proportional to $T^{2}$ at high temperatures as in the classical theory and contain zero-point energy contributions at low temperatures, a quantum effect. All they contribute to is getting the accurate information on structural and thermodynamic parameters of fcc crystals taken from XAFS experiments. The good agreement of the above calculated results with the experiment makes it possible to reproduce the experimental XAFS data of fcc crystals using the present theory. Moreover, this agreement also shows that the FSNNCA is sufficient to take into account the manybody effects in the present one-dimensional model applied to XAFS studies of the thermodynamic properties and anharmonic effects of the considered crystals at least for $\mathrm{Cu}$ and $\mathrm{Ni}$.

\section{Conclusions}

In this work, the thermodynamic properties and anharmonic effects of materials have been studied based on XAFS DWFs presented in terms of cumulant expansion up to the third order which includes dispersion relation containing more information on atomic vibrations taken from integration over the first BZ. This theory can be applied to any crystal structure including complex systems.

The ACDM applied to this work has been derived using the MBPA based on the dualism (corpuscular and wave property) of an elementary particle in quantum theory to describe the system involving all different frequencies up to Debye frequency as a system consisting of many phonons, as well as the FSNNCA to take into account the many-body effects in the present one-dimensional model. Based on these approaches, the derivations of the analytical expressions and the numerical calculations of XAFS quantities have been significantly simplified and reduced.

The derived analytical expressions of three first XAFS cumulants satisfy all their fundamental properties in temperature dependence and contribute to the valuation of the thermodynamic properties and anharmonic effect in XAFS of the considered materials where they approach the classical values at high temperature and contain zero-point energy contributions at low temperatures, a quantum effect. All they contribute to is getting the accurate information on structural and thermodynamic parameters of the considered materials taken from XAFS experiments.

The good agreement of numerical results for $\mathrm{Cu}$ and $\mathrm{Ni}$ with experiment and with those calculated using the other theories illustrates the advantages and efficiency of the present theory in the calculation and analysis of the thermodynamic properties and anharmonic effects in XAFS of substances and in material studies.

\section{Conflicts of Interest}

The authors declare that they have no conflicts of interest.

\section{Acknowledgments}

The authors thank Professor J. J. Rehr and Professor P. Fornasini for useful comments.

\section{References}

[1] E. D. Crozier, J. J. Rehr, and R. Ingalls, in X-ray Absorption, D. C. Koningsberger and R. Prins, Eds., Chapter 9, Wiley, Wiley, New York, NY, USA, 1988.

[2] M. Tranquada and R. Ingalls, "Extended x-ray-absorption fine-structure study of anharmonicity in CuBr," Physical Review B, vol. 28, no. 6, pp. 3520-3528, 1983.

[3] N. V. Hung and R. Frahm, "Temperature and shell size dependence of anharmonicity in EXAFS," Physica B: Condensed Matter, vol. 208-209, pp. 97-99, 1995.

[4] N. V. Hung, R. Frahm, and H. Kamitsubo, "Anharmonic contributions to high-temperature EXAFS spectra: theory and comparison with experiment," Journal of the Physical Society of Japan, vol. 65, no. 11, pp. 3571-3575, 1996.

[5] E. A. Stern, P. Livins, and Z. Zhang, "Thermal vibration and melting from a local perspective," Physical Review B, vol. 43, no. 11, pp. 8850-8860, 1991.

[6] N. V. Hung, T. S. Tien, N. B. Duc, and D. Q. Vuong, "Highorder expanded XAFS Debye-Waller factors of HCP crystals based on classical anharmonic correlated Einstein model," Modern Physics Letters B, vol. 28, no. 21, article 1450174, 2014.

[7] A. I. Frenkel and J. J. Rehr, "Thermal expansion and x-rayabsorption fine-structure cumulants," Physical Review B, vol. 48, no. 1, pp. 585-588, 1993.

[8] T. Miyanaga and T. Fujikawa, "Quantum statistical approach to Debye-Waller factor in EXAFS, EELS and ARXPS. II. Application to one-dimensional models," Journal of the Physical Society of Japan, vol. 63, no. 3, pp. 1036-1052, 1994.

[9] N. V. Hung and J. J. Rehr, "Anharmonic correlated Einsteinmodel Debye-Waller factors," Physical Review B, vol. 56, no. 1, pp. 43-46, 1997.

[10] N. V. Hung, N. B. Duc, and R. R. Frahm, "A new anharmonic factor and EXAFS including anharmonic contributions," Journal of the Physical Society of Japan, vol. 72, no. 5, pp. 1254-1259, 2003.

[11] M. Daniel, D. M. Pease, N. V. Hung, and J. I. Budnick, "Local force constants of transition metal dopants in a nickel host: comparison to Mossbauer studies," Physical Review B, vol. 69, no. 13, article 134414, 2004.

[12] N. V. Hung and P. Fornasini, "Anharmonic effective potential, correlation effects, and EXAFS cumulants calculated from a Morse interaction potential for fcc metals," Journal of the Physical Society of Japan, vol. 76, no. 8, article 084601, 2007.

[13] N. V. Hung, C. S. Thang, N. B. Duc, D. Q. Vuong, and T. S. Tien, "Advances in theoretical and experimental XAFS studies of thermodynamic properties, anharmonic effects and structural determination of fcc crystals," European Physical Journal B, vol. 90, no. 12, p. 256, 2017.

[14] N. V. Hung, T. S. Tien, L. H. Hung, and R. R. Frahm, "Anharmonic effective potential, local force constant and EXAFS of HCP crystals: theory and comparison to experiment," International Journal of Modern Physics B, vol. 22, no. 29, pp. 5155-5166, 2008.

[15] N. V. Hung, "Pressure-dependent anharmonic correlated einstein model extended X-ray absorption fine structure Debye-Waller Factors," Journal of the Physical Society of Japan, vol. 83, no. 2, article 024802, 2014.

[16] V. V. Hung, H. K. Hieu, and K. Masuda-Jindo, "Study of EXAFS cumulants of crystals by the statistical moment method and anharmonic correlated Einstein model," Computational Materials Science, vol. 49, no. 4, pp. S214-S217, 2010. 
[17] N. V. Hung, C. S. Thang, N. C. Toan, and H. K. Hieu, "Temperature dependence of Debye-Waller factors of semiconductors," Vacuum, vol. 101, pp. 63-66, 2014.

[18] T. Yokoyama, "Path-integral effective-potential method applied to extended x-ray-absorption fine-structure cumulants," Physical Review B, vol. 57, pp. 3423-3432, 1998.

[19] S. A. Beccara, G. Dalba, P. Fornasini, R. Grisenti, F. Pederiva, and A. Sanson, "Local thermal expansion in copper: extended $\mathrm{x}$-ray-absorption fine-structure measurements and pathintegral Monte Carlo calculations," Physical Review B, vol. 68, no. 14, article 140301(R), 2003.

[20] E. Sevillano, M. Meuth, and J. J. Rehr, "Extended x-ray absorption fine structure Debye-Waller factors. I. Monatomic crystals," Physical Review B, vol. 20, no. 12, pp. 4908-4911, 1979.

[21] F. D. Vila, J. J. Rehr, H. H. Rossner, and H. J. Krappe, “Theoretical x-ray absorption Debye-Waller factors," Physical Review B, vol. 76, no. 1, article 014301, 2007.

[22] R. B. Greegor and F. W. Lytle, "Extended x-ray absorption fine structure determination of thermal disorder in $\mathrm{Cu}$ : comparison of theory and experiment," Physical Review B, vol. 20, no. 12 , pp. 4902-4907, 1979.

[23] V. Pirog, T. I. Nedoseikina, A. I. Zarubin, and A. T. Shuvaev, "Anharmonic pair potential study in face-centred-cubic structure metals," Journal of Physics: Condensed Matter, vol. 14, no. 8, pp. 1825-1832, 2002.

[24] T. Yokoyama, T. Sasukawa, and T. Ohta, "Anharmonic interatomic potentials of metals and metal bromides determined by EXAFS," Japanese Journal of Applied Physics, vol. 28, no. 10, pp. 1905-1908, 1989.

[25] P. Fornasini, S. A. Beccara, G. Dalba, R. Grisenti, A. Sanson, and M. Vaccari, "Extended x-ray-absorption fine-structure measurements of copper: local dynamics, anharmonicity, and thermal expansion," Physical Review B, vol. 70, no. 17, article 174301, 2004.

[26] G. Dalba, P. Fornasini, R. Grisenti, and J. Purans, "Sensitivity of extended X-ray-absorption fine structure to thermal expansion," Physical Review Letters, vol. 82, no. 21, pp. 42404243, 1999.

[27] N. V. Hung, N. B. Trung, and B. Kirchner, "Anharmonic correlated Debye model Debye-Waller factors," Physica B: Condensed Matter, vol. 405, no. 11, pp. 2519-2525, 2010.

[28] N. V. Hung, T. T. Hue, H. D. Khoa, and D. Q. Vuong, "Anharmonic correlated Debye model high-order expanded interatomic effective potential and Debye-Waller factors of bcc crystals," Physica B: Condensed Matter, vol. 503, pp. 174-178, 2016.

[29] G. D. Mahan, Many-Particle Physics, Plenum, New York, NY, USA, 2nd edition, 1990 .

[30] G. K. Horton and A. A. Maradudin, Dynamical Properties of Solids, Vol. 1, North Holland, Amsterdam, Netherlands, 1974.

[31] L. A. Girifalco and W. G. Weizer, "Application of the morse potential function to cubic metals," Physical Review, vol. 114, no. 3, pp. 687-690, 1959.

[32] P. Eisenberger and G. S. Brown, "The study of disordered systems by EXAFS: limitations," Solid State Communications, vol. 29, no. 6, pp. 481-484, 1979.

[33] J. B. Boyce, T. M. Hayes, and J. C. Mikkensen Jr., "Extended-xray-absorption-fine-structure investigation of mobile-ion density in superionic AgI, CuI, CuBr, and $\mathrm{CuCl}$," Physical Review B, vol. 23, no. 6, pp. 2876-2896, 1981.

[34] T. Yokoyama, "Path-integral effective-potential theory for EXAFS cumulants compared with the second-order perturbation," Journal of Synchrotron Radiation, vol. 6, no. 3, pp. 323-325, 1999.

[35] L. Tröger, T. Yokoyama, T. Arvanitis, T. Lederer, M. Tischer, and $\mathrm{K}$. Baberschke, "Determination of bond lengths, atomic mean-square relative displacements, and local thermal expansion by means of soft-x-ray photoabsorption," Physical Review B, vol. 49, no. 2, pp. 888-903, 1994.

[36] G. Dalba, P. Fornasini, R. Gotter, and F. Rocca, "Anharmonicity effects on the extended $x$-ray-absorption fine structure: the case of $\beta$-AgI," Physical Review B, vol. 52, no. 1 , pp. 149-157, 1995.

[37] B. T. M. Wilis and A. W. Pryor, Thermal Vibrations in Crystallography, Cambridge University Press, Cambridge, UK, 1975.

[38] T. Ishii, "Note on the $\mathrm{K}$ extended X-ray absorption finestructure Debye-Waller factor," Journal of Physics: Condensed Matter, vol. 4, no. 40, pp. 8029-8034, 1992. 


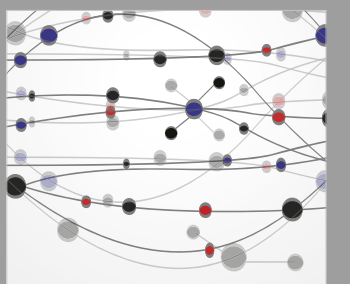

The Scientific World Journal
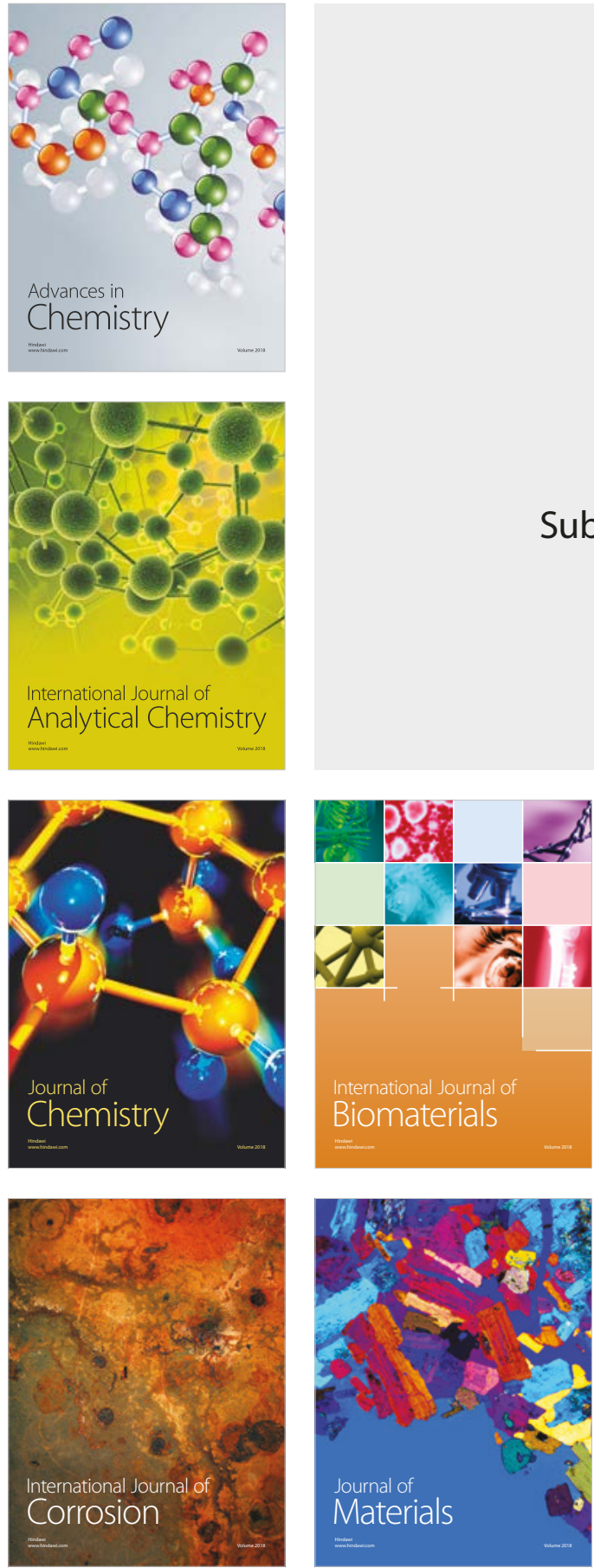

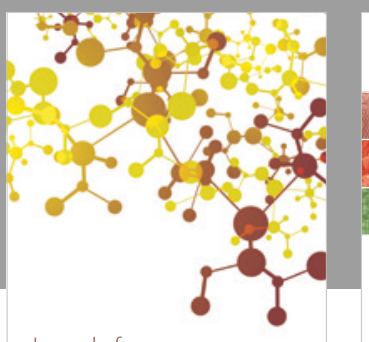

Journal of

Applied Chemistry
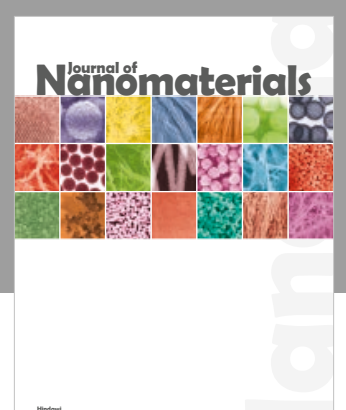

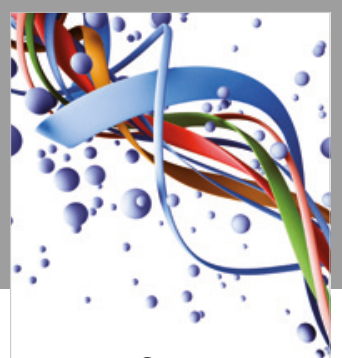

Scientifica

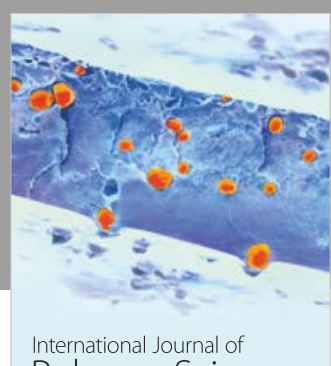

Polymer Science

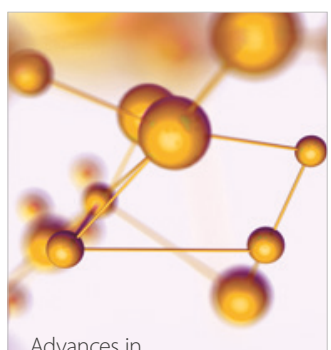

Physical Chemistry
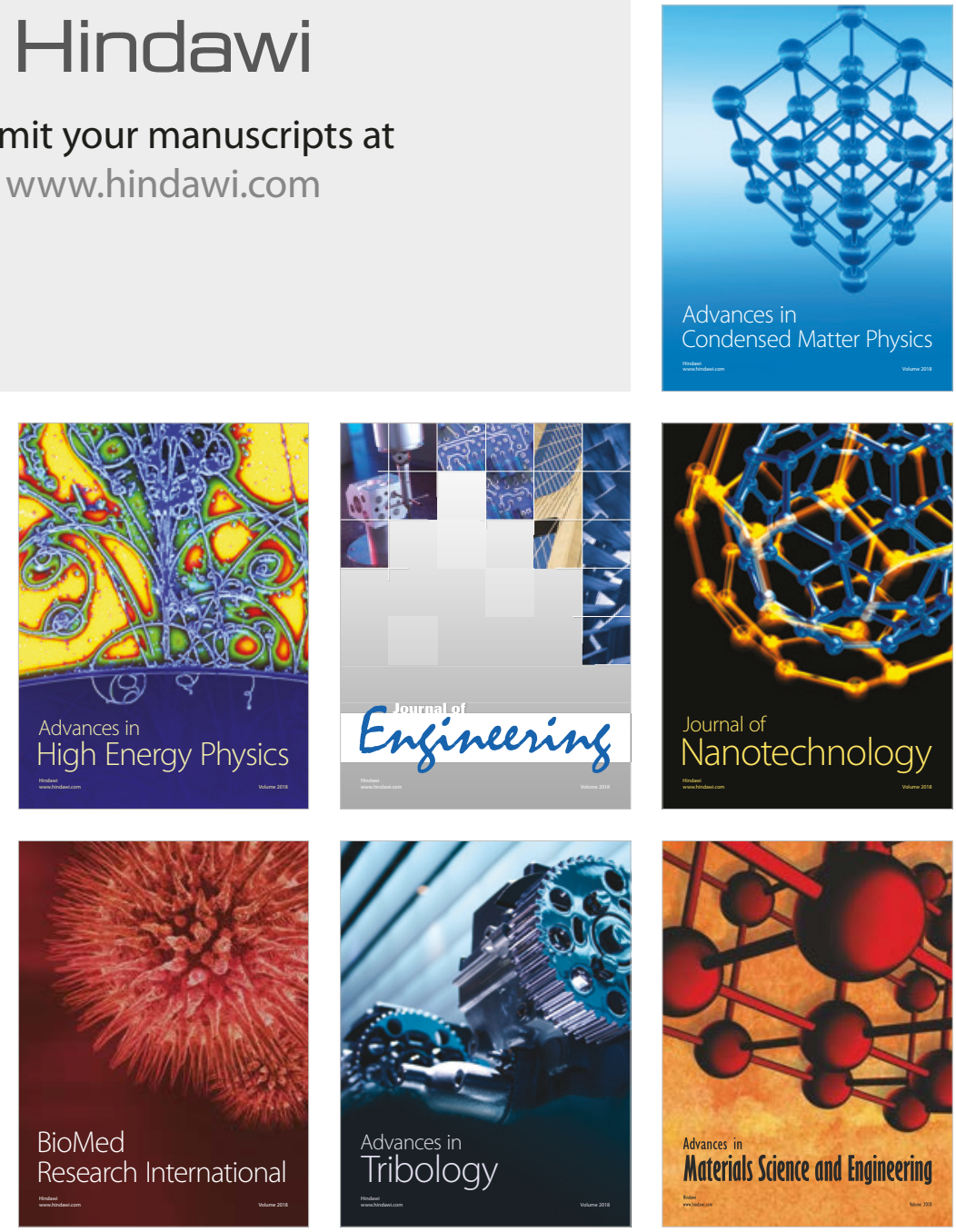